\title{
HILBERT-SAMUEL MULTIPLICITIES OF CERTAIN DEFORMATION RINGS
}

\author{
FABIAN SANDER
}

\begin{abstract}
We compute presentations of crystalline framed deformation rings of a twodimensional representation $\bar{\rho}$ of the absolute Galois group of $\mathbb{Q}_{p}$, when $\bar{\rho}$ has scalar semi-simplification, the Hodge-Tate weights are small and $p>2$. In the non-trivial cases, we show that the special fibre is geometrically irreducible, generically reduced and the Hilbert-Samuel multiplicity is either 1, 2 or 4 depending on $\bar{\rho}$. We show that in the last two cases the deformation ring is not Cohen-Macaulay.
\end{abstract}

\section{Introduction}

Let $p>2$ be a prime. Let $k$ be a finite field of characteristic $p, E$ be a finite totally ramified extension of $W(k)\left[\frac{1}{p}\right]$ with ring of integers $\mathcal{O}$ and uniformizer $\pi$. For a given continuous representation $\bar{\rho}: G_{\mathbb{Q}_{p}} \rightarrow \mathrm{GL}_{2}(k)$ we consider the universal framed deformation ring $R_{\bar{\rho}}^{\square}$ and the universal framed deformation $\rho^{\text {univ }}: G_{\mathbb{Q}_{p}} \rightarrow \operatorname{GL}_{2}\left(R_{\bar{\rho}}^{\square}\right)$. For all $\mathfrak{p} \in \mathrm{m}-\operatorname{Spec}\left(R_{\bar{\rho}}^{\square}\left[\frac{1}{p}\right]\right)$, the set of maximal ideals of $R_{\bar{\rho}}^{\square}\left[\frac{1}{p}\right]$, we can specialize the universal representation at $\mathfrak{p}$ to obtain the representation

$$
\rho_{\mathfrak{p}}: G_{\mathbb{Q}_{p}} \rightarrow \mathrm{GL}_{2}\left(R_{\bar{\rho}}^{\square}\left[\frac{1}{p}\right] / \mathfrak{p}\right),
$$

where $R_{\bar{\rho}}^{\square}\left[\frac{1}{p}\right] / \mathfrak{p}$ is a finite extension of $\mathbb{Q}_{p}$. Let $\tau: I_{G_{\mathbb{Q}_{p}}} \rightarrow \mathrm{GL}_{2}(E)$ be a representation with an open kernel, where $I_{G_{\mathbb{Q}_{p}}}$ is the inertia subgroup of $G_{\mathbb{Q}_{p}}$. We also fix integers $a, b$ with $b \geq 0$ and a continuous character $\psi: G_{\mathbb{Q}_{p}} \rightarrow \mathcal{O}^{\times}$such that $\overline{\psi \epsilon}=\operatorname{det}(\bar{\rho})$, where $\epsilon$ is the cyclotomic character. Kisin showed in [10] that there exist unique reduced $\mathcal{O}$-torsion free quotients $R_{\bar{\rho}}^{\square, \psi}(a, b, \tau)$ and $R_{\bar{\rho}, \text {,cris }}^{\square, \psi}(a, b, \tau)$ of $R_{\bar{\rho}}^{\square}$ with the property that $\rho_{\mathfrak{p}}$ factors through $R_{\bar{\rho}}^{\square, \psi}(a, b, \tau)$ resp. $R_{\bar{\rho}, \text { cris }}^{\square, \psi}(a, b, \tau)$ if and only if $\rho_{\mathfrak{p}}$ is potentially semi-stable resp. potentially crystalline with Hodge-Tate weights $(a, a+b+1)$ and has determinant $\psi \epsilon$ and inertial type $\tau$. If $\tau$ is trivial then $R_{\bar{\rho}, \text { cris }}^{\square, \psi}(a, b):=R_{\bar{\rho}, \text { cris }}^{\square, \psi}(a, b, \mathbb{1} \oplus \mathbb{1})$ parametrizes all the crystalline lifts of $\bar{\rho}$ with Hodge-Tate weights $(a, a+b+1)$ and determinant $\psi \epsilon$. The Breuil-Mézard conjecture, proved by Kisin for almost all $\bar{\rho}$, see also $[2,3,7,8,14]$, says that the Hilbert-Samuel multiplicity of the $\operatorname{ring} R_{\bar{\rho}}^{\square, \psi}(a, b, \tau) / \pi$ can be determined by computing certain automorphic multiplicities, which do not depend on $\bar{\rho}$, and the Hilbert-Samuel multiplicities of $R_{\bar{\rho}, \text { cris }}^{\square, ~}(a, b)$ in low weights for $0 \leq a \leq p-2,0 \leq b \leq p-1$. For most $\bar{\rho}$, the Hilbert-Samuel multiplicities of $R_{\bar{\rho}, \text { cris }}^{\square, \psi}(a, b)$ have already been determined. Our goal in this paper is to compute the

Received by the editors December 20, 2012. 
Hilbert-Samuel multiplicity of the ring $R_{\bar{\rho}, \text { cris }}^{\square, \psi}(a, b)$ with $0 \leq a \leq p-2,0 \leq b \leq p-1$ when

$$
\bar{\rho}: G_{\mathbb{Q}_{p}} \rightarrow \mathrm{GL}_{2}(k), \quad g \mapsto\left(\begin{array}{cc}
\chi(g) & \phi(g) \\
0 & \chi(g)
\end{array}\right) .
$$

One may show that $R_{\bar{\rho}, \text { cris }}^{\square, \psi}(a, b)$ is zero if either $b \neq p-2$ or the restriction of $\chi$ to $I_{\mathbb{Q}_{p}}$ is not equal to $\epsilon^{a}$ modulo $\pi$.

Theorem 1. Let $a$ be an integer with $0 \leq a \leq p-2$ such that $\left.\chi\right|_{\mathbb{Q}_{p}} \equiv \epsilon^{a}(\bmod \pi)$. Then $R_{\bar{\rho}, \mathrm{cris}}^{\square, \psi}(a, p-2) / \pi$ is geometrically irreducible, generically reduced and

$$
e\left(R_{\bar{\rho}, \text { cris }}^{\square, \psi}(a, p-2) / \pi\right)=\left\{\begin{array}{l}
1 \text { if } \bar{\rho} \otimes \chi^{-1} \text { is ramified, } \\
2 \text { if } \bar{\rho} \otimes \chi^{-1} \text { is unramified, indecomposable } \\
4 \text { if } \bar{\rho} \otimes \chi^{-1} \text { is split. }
\end{array}\right.
$$

In the last two cases, $R_{\bar{\rho}, \text { cris }}^{\square, \psi}(a, p-2)$ is not Cohen-Macaulay.

The multiplicity 4 does not seem to have been anticipated in the literature, see for example $[11,1.1 .6]$. Our method is elementary in the sense that we do not use any integral $p$-adic Hodge theory. The only $p$-adic Hodge theoretic input is that if $\rho$ is a crystalline lift of $\bar{\rho}$ with Hodge-Tate weights $(0, p-1)$, then we have an exact sequence

$$
0 \longrightarrow \epsilon^{p-1} \chi_{1} \longrightarrow \rho \longrightarrow \chi_{2} \longrightarrow 0,
$$

where $\chi_{1}, \chi_{2}: G_{\mathbb{Q}_{p}} \rightarrow \mathcal{O}^{\times}$are unramified characters. This allows us to convert the problem into a linear algebra problem, which we solve in Lemma 2. This gives us an explicit presentation of the ring $R_{\bar{\rho}, \text { cris }}^{\square,}(a, p-2)$, using which we compute the multiplicities in Section 4. Our argument gives a proof of the existence of $R_{\bar{\rho}, \text { cris }}^{\square \cdot \psi}(a, p-$ 2 ) independent of [10]. After writing this note we discovered that the idea to convert the problem into linear algebra already appears in [15].

\section{The universal ring}

After twisting we may assume that $\chi=1$ and $a=0$ so that

$$
\bar{\rho}(g)=\left(\begin{array}{cc}
1 & \phi(g) \\
0 & 1
\end{array}\right) .
$$

Since the image of $\bar{\rho}$ in $\mathrm{GL}_{2}(k)$ is a $p$-group, the universal representation factors through the maximal pro- $p$ quotient of $G_{\mathbb{Q}_{p}}$, which we denote by $G$. We have the following commuting diagram:

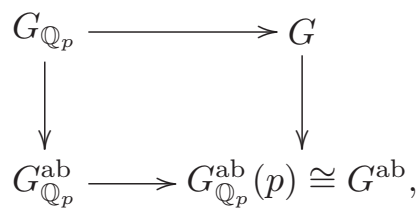


where $G_{\mathbb{Q}_{p}}^{\mathrm{ab}}:=\operatorname{Gal}\left(\mathbb{Q}_{p}^{\mathrm{ab}} / \mathbb{Q}_{p}\right)$ is the maximal abelian quotient of $G_{\mathbb{Q}_{p}}$ and can be described by the exact sequence

$$
1 \longrightarrow \operatorname{Gal}\left(\mathbb{Q}_{p}^{\mathrm{ab}} / \mathbb{Q}_{p}^{\mathrm{ur}}\right) \longrightarrow G_{\mathbb{Q}_{p}}^{\mathrm{ab}} \longrightarrow G_{\mathbb{F}_{p}} \longrightarrow 1
$$

where $\mathbb{Q}_{p}^{\text {ur }}$ is the maximal unramified extension of $\mathbb{Q}_{p}$ inside $\overline{\mathbb{Q}}_{p}$. Local class field theory implies that the natural map

$$
G_{\mathbb{Q}_{p}}^{\mathrm{ab}} \rightarrow \operatorname{Gal}\left(\mathbb{Q}_{p}^{\text {ur }} / \mathbb{Q}_{p}\right) \times \operatorname{Gal}\left(\mathbb{Q}_{p}\left(\mu_{p} \infty\right) / \mathbb{Q}_{p}\right)
$$

is an isomorphism, where $\mu_{p}$ is the group of $p$-power order roots of unity in $\overline{\mathbb{Q}}_{p}$. The cyclotomic character $\epsilon$ induces an isomorphism

$$
\operatorname{Gal}\left(\mathbb{Q}_{p}\left(\mu_{p}\right) / \mathbb{Q}_{p}\right) \stackrel{\cong}{\stackrel{\cong}{\longrightarrow}} \mathbb{Z}_{p}^{\times}
$$

and $\operatorname{Gal}\left(\mathbb{Q}_{p}^{\text {ur }} / \mathbb{Q}_{p}\right) \cong \hat{\mathbb{Z}}$, hence

$$
G^{\mathrm{ab}} \cong\left(1+p \mathbb{Z}_{p}\right) \times \mathbb{Z}_{p}
$$

where the map onto the first factor is given by $\epsilon^{p-1}$. We choose a pair of generators $\bar{\gamma}, \bar{\delta}$ of $G^{\text {ab }}$ such that $\bar{\gamma} \mapsto(1+p, 0)$ and $\bar{\delta} \mapsto(1,1)$. With [1, Lemma 3.2] we obtain that $G$ is a free pro- $p$ group in two letters $\gamma, \delta$ which project to $\bar{\gamma}, \bar{\delta}$. The way we choose these generators will be of importance in the following.

Lemma 1. Let $\eta: G_{\mathbb{Q}_{p}} \rightarrow \mathbb{Z}_{p}^{\times}$be a continuous character such that $\eta \equiv 1(p)$. Then $\eta=\epsilon^{k} \chi$ for an unramified character $\chi$ if and only if $\eta(\gamma)=\epsilon(\gamma)^{k}$ and $p-1 \mid k$.

Proof. " $\Rightarrow$ : " Since $\gamma$ maps to identity in $\operatorname{Gal}\left(\mathbb{Q}_{p}^{\text {ur }} / \mathbb{Q}_{p}\right)$, we clearly have $\chi(\gamma)=1$ for every unramified character $\chi$. Hence $\epsilon(\gamma)^{k} \equiv 1(p)$, which implies $p-1 \mid k$.

" $\Leftarrow$ : " From $\eta \epsilon^{-k}(\gamma)=1$ and the fact that $\delta$ maps to the image of identity in the maximal pro- $p$ quotient of $\operatorname{Gal}\left(\mathbb{Q}_{p}\left(\mu_{p} \infty\right) / \mathbb{Q}_{p}\right)$, we see that $\eta \epsilon^{-k}=\chi$ for an unramified character $\chi$.

Since $G$ is a free pro- $p$ group generated by $\gamma$ and $\delta$, to give a framed deformation of $\bar{\rho}$ to $\left(A, \mathfrak{m}_{A}\right)$ is equivalent to give two matrices in $\operatorname{GL}_{2}(A)$ which reduce to $\bar{\rho}(\gamma)$ and $\bar{\rho}(\delta)$ modulo $\mathfrak{m}_{A}$. Thus

$$
R_{\bar{\rho}}^{\square}=\mathcal{O}\left[\left[x_{11}, \hat{x}_{12}, x_{21}, t_{\gamma}, y_{11}, \hat{y}_{12}, y_{21}, t_{\delta}\right]\right]
$$

and the universal framed deformation is given by

$$
\begin{gathered}
\rho^{\text {univ }}: G \rightarrow \mathrm{GL}_{2}\left(R_{\bar{\rho}}^{\square}\right), \\
\gamma \mapsto\left(\begin{array}{cc}
1+t_{\gamma}+x_{11} & x_{12} \\
x_{21} & 1+t_{\gamma}-x_{11}
\end{array}\right), \\
\delta \mapsto\left(\begin{array}{cc}
1+t_{\delta}+y_{11} & y_{12} \\
y_{21} & 1+t_{\delta}-y_{11}
\end{array}\right),
\end{gathered}
$$

where $x_{12}:=\hat{x}_{12}+[\phi(\gamma)], y_{12}:=\hat{y}_{12}+[\phi(\delta)]$ where $[\phi(\gamma)],[\phi(\delta)]$ denote the Teichmüller lifts of $\phi(\gamma)$ and $\phi(\delta)$ to $\mathcal{O}$. 
Remark 1. We note that there are essentially three different cases:

(1) $\bar{\rho}$ is ramified $\Leftrightarrow \phi(\gamma) \neq 0 \Leftrightarrow x_{12} \in\left(R_{\bar{\rho}}^{\square}\right)^{\times}$;

(2) $\bar{\rho}$ is unramified, non-split $\Leftrightarrow \phi(\gamma)=0, \phi(\delta) \neq 0 \Leftrightarrow x_{12} \in \mathfrak{m}_{R_{\bar{\rho}}}, y_{12} \in\left(R_{\bar{\rho}}^{\square}\right)^{\times}$;

(3) $\bar{\rho}$ is split $\Leftrightarrow \phi(\gamma)=0, \phi(\delta)=0 \Leftrightarrow x_{12}, y_{12} \in \mathfrak{m}_{R_{\bar{\rho}}}$.

Let $\psi: G_{\mathbb{Q}_{p}} \rightarrow \mathcal{O}^{\times}$be a continuous character, such that $\operatorname{det}(\bar{\rho})=\overline{\psi \epsilon}$, and let $R_{\bar{\rho}}^{\square, \psi}$ be the quotient of $R_{\bar{\rho}}^{\square}$ which parametrizes lifts of $\bar{\rho}$ with determinant $\psi \epsilon$. Since $\gamma, \delta$ generate $G$ as a group, we obtain

$$
\begin{aligned}
R_{\bar{\rho}}^{\square, \psi} & \cong R_{\bar{\rho}}^{\square} /\left(\operatorname{det}\left(\rho^{\mathrm{univ}}(\gamma)-\psi \epsilon(\gamma)\right), \operatorname{det}\left(\rho^{\mathrm{univ}}(\delta)-\psi \epsilon(\delta)\right)\right) \\
& \cong \mathcal{O}\left[\left[x_{11}, \hat{x}_{12}, x_{21}, y_{11}, \hat{y}_{12}, y_{21}\right]\right],
\end{aligned}
$$

because we can eliminate the parameters $t_{\gamma}, t_{\delta}$ due to the relations $\left(1+t_{\gamma}\right)^{2}=\psi \epsilon(\gamma)+$ $x_{11}^{2}+x_{12} x_{21}, t_{\gamma} \equiv 0(\mathfrak{m}),\left(1+t_{\delta}\right)^{2}=\psi \epsilon(\delta)+y_{11}^{2}+y_{12} y_{21}, t_{\delta} \equiv 0(\mathfrak{m})$. We let $v:=$ $\frac{1-\epsilon^{p-1}(\gamma)}{2}$ and define four polynomials

$$
\begin{aligned}
& I_{1}:=\left(v+x_{11}\right)\left(v-x_{11}\right)-x_{12} x_{21} \\
& I_{2}:=\left(v+x_{11}\right)^{2} y_{12}-2\left(v+x_{11}\right) x_{12} y_{11}-x_{12}^{2} y_{21}, \\
& I_{3}:=x_{21}^{2} y_{12}-2 x_{21}\left(v-x_{11}\right) y_{11}-\left(v-x_{11}\right)^{2} y_{21}, \\
& I_{4}:=\left(v+x_{11}\right) x_{21} y_{12}-2 x_{12} x_{21} y_{11}-x_{12}\left(v-x_{11}\right) y_{21} .
\end{aligned}
$$

Since for every representation with Hodge-Tate weights $(0, p-1)$ the determinant is a character of Hodge-Tate weight $p-1$ and $R_{\bar{\rho}, \text { cris }}^{\square, \psi}(0, p-2)$ parametrizes all lifts $\rho_{\mathfrak{p}}$ with determinant $\psi \epsilon$, we let from now on $\psi$ have Hodge-Tate weight $p-2$, as otherwise $R_{\bar{\rho}, \text { cris }}^{\square, \psi}(0, p-2)$ would be trivial.

Definition 1. We set

$$
R:=R_{\bar{\rho}}^{\square, \psi} /\left(I_{1}, I_{2}, I_{3}, I_{4}\right) .
$$

Our goal is to show that $R_{\bar{\rho}, \text { cris }}^{\square, \psi}(0, p-2)$ is isomorphic to $R$.

Lemma 2. If $\mathfrak{p} \in \mathrm{m}-\operatorname{Spec}\left(R_{\bar{\rho}}^{\square, \psi}\left[\frac{1}{p}\right]\right)$, then $\mathfrak{p} \in \mathrm{m}-\operatorname{Spec}\left(R\left[\frac{1}{p}\right]\right)$ if and only if $\rho_{\mathfrak{p}}$ is reducible and $\rho_{\mathfrak{p}}(\gamma)$ acts on the $G$-invariant subspace with eigenvalue $\epsilon^{p-1}(\gamma)$.

Proof. Let $\mathfrak{p} \in \mathrm{m}-\operatorname{Spec}\left(R_{\bar{\rho}}^{\square, \psi}\left[\frac{1}{p}\right]\right)$, such that $\rho_{\mathfrak{p}}$ is reducible and $\rho_{\mathfrak{p}}(\gamma)$ acts on the $G$ invariant subspace with eigenvalue $\epsilon^{p-1}(\gamma)$. Since $\operatorname{det}\left(\rho_{\mathfrak{p}}(\gamma)\right)=\psi \epsilon(\gamma)=\epsilon(\gamma)^{p-1}$ and $\epsilon(\gamma)^{p-1}$ is an eigenvalue of $\rho_{\mathfrak{p}}(\gamma)$, the other eigenvalue must be 1 . Therefore we can write $1+t_{\gamma}=\frac{\epsilon(\gamma)^{p-1}+1}{2}$ and obtain

$$
\begin{aligned}
0 & =\operatorname{det}\left(\begin{array}{cc}
1+t_{\gamma}+x_{11}-\epsilon(\gamma)^{p-1} & x_{12} \\
x_{21} & 1+t_{\gamma}-x_{11}-\epsilon(\gamma)^{p-1}
\end{array}\right) \\
& =\left(v+x_{11}\right)\left(v-x_{11}\right)-x_{12} x_{21} .
\end{aligned}
$$


If we now take $\mathfrak{p}$ as above but with $I_{1}:=\left(v+x_{11}\right)\left(v-x_{11}\right)-x_{12} x_{21} \in \mathfrak{p}$, it is easy to see that the vectors $v_{1}=\left(\begin{array}{c}-x_{12} \\ v+x_{11}\end{array}\right)$ and $v_{2}=\left(\begin{array}{c}v-x_{11} \\ -x_{21}\end{array}\right)$ are eigenvectors for $\rho_{\mathfrak{p}}(\gamma)$ with eigenvalue $\epsilon(\gamma)^{p-1}$ if they are non-zero. But at least one of them is non-zero because otherwise we obtain $v=0$ and thus $\epsilon(\gamma)^{p-1}=1$, which is a contradiction to the definition of $\gamma$. So $\rho_{\mathfrak{p}}$ is reducible with an invariant subspace on which $\rho_{\mathfrak{p}}(\gamma)$ acts by $\epsilon(\gamma)^{p-1}$ if and only if the vectors $v_{1}, v_{2}, \rho^{\text {univ }}(\delta) v_{1}, \rho^{\text {univ }}(\delta) v_{2}$ are pairwise linear dependent. It is easy to check that this is equivalent to the satisfaction of the equations $I_{1}=I_{2}=I_{3}=I_{4}=0$.

\section{Lemma 3.}

$$
\mathrm{m}-\operatorname{Spec}\left(R\left[\frac{1}{p}\right]\right)=\mathrm{m}-\operatorname{Spec}\left(R_{\bar{\rho}}^{\square, \psi}(0, p-2)\left[\frac{1}{p}\right]\right) .
$$

Proof. From Khare and Wintenberger [9, Proposition 3.5(i)] we know that every crystalline lift $\rho_{\mathfrak{p}}$ of a reducible two-dimensional representation $\bar{\rho}$, such that $\rho_{\mathfrak{p}}$ has Hodge-Tate-weights $(0, p-1)$, is reducible itself. Moreover, Brinon and Conrad [4, Theorem 8.3.5] say that if $\rho$ is a reducible two-dimensional crystalline representation, then we have an exact sequence

$$
0 \longrightarrow \epsilon^{p-1} \chi_{1} \longrightarrow \rho \longrightarrow \chi_{2} \longrightarrow 0 \text {. }
$$

Thus $\rho_{\mathfrak{p}}(\gamma)$ acts on the invariant subspace as $\epsilon(\gamma)^{p-1}$ and hence from Lemma 2 it is clear that

$$
\mathrm{m}-\operatorname{Spec}\left(R\left[\frac{1}{p}\right]\right) \supset \mathrm{m}-\operatorname{Spec}\left(R_{\bar{\rho}}^{\square, \psi}(0, p-2)\left[\frac{1}{p}\right]\right) .
$$

For the other inclusion we note that it is also clear from Lemma 2 that any maximal ideal $\mathfrak{p} \in \mathrm{m}-\operatorname{Spec}\left(R\left[\frac{1}{p}\right]\right)$ gives rise to a reducible representation $\rho_{\mathfrak{p}}$ such that $\rho_{\mathfrak{p}}(\gamma)$ acts on the invariant subspace as $\epsilon(\gamma)^{p-1}$ and that the other eigenvalue of $\rho_{\mathfrak{p}}(\gamma)$ is 1 . So we obtain with Lemma 1 that $\rho_{\mathfrak{p}}$ is an extension of two crystalline characters

$$
0 \rightarrow \eta_{1} \rightarrow * \rightarrow \eta_{2} \rightarrow 0
$$

where the Hodge-Tate weight of $\eta_{1}$ is equal to $p-1$ and the weight of $\eta_{2}$ is equal to 0. Then we can conclude from [13, Proposition 128] that it is semi-stable and from $[4$, Theorem 8.3.5, Proposition 8.38] that it is crystalline and hence $\mathfrak{p} \in \mathrm{m}$-Spec $\left(R_{\bar{\rho}}^{\square, \psi}(0, p-2)\left[\frac{1}{p}\right]\right)$.

Remark 2. We have the following identities $\bmod I_{1}$ :

$$
\begin{aligned}
x_{21} I_{2} & =\left(v+x_{11}\right) I_{4}, \\
\left(v-x_{11}\right) I_{2} & =x_{12} I_{4}, \\
x_{21} I_{4} & =\left(v+x_{11}\right) I_{3}, \\
\left(v-x_{11}\right) I_{4} & =x_{12} I_{3} .
\end{aligned}
$$




\section{Reducedness}

In order to show that $R_{\bar{\rho}}^{\square, \psi}(0, p-2)$ is equal to $R$, it is enough to show that $R$ is reduced and $\mathcal{O}$-torsion free, since then the assertion follows from Lemma 3, as $R\left[\frac{1}{p}\right]$ is Jacobson because $R$ is a quotient of a formal power series ring over a complete discrete valuation ring.

Lemma 4. If $\mathcal{O}=W(k)$, then $R$ is an $W(k)$-torsion-free integral domain.

Proof. We distinguish two cases.

If $\bar{\rho}$ is ramified, i.e., $x_{12}$ is invertible, we consider the fact that for every complete local ring $A$ with $a \in \mathfrak{m}_{A}, u \in A^{\times}$, there is a canonical isomorphism $A[[z]] /(u z-a) \cong$ $A$. Using this we see from (1),(2),(6) and (8) that

$$
\begin{aligned}
R & =\mathcal{O}\left[\left[x_{11}, \hat{x}_{12}, x_{21}, y_{11}, \hat{y}_{12}, y_{21}\right]\right] /\left(I_{1}, I_{2}\right) \\
& \cong \mathcal{O}\left[\left[x_{11}, \hat{x}_{12}, y_{11}, \hat{y}_{12}\right]\right]
\end{aligned}
$$

which shows the claim.

In the second case, where $\bar{\rho}$ is unramified, i.e., $x_{12} \notin R^{\times}$, we consider the ideal $I:=\left(\pi, x_{11}, x_{12}, x_{21}\right)$ and have

$$
\operatorname{gr}_{I} R_{\bar{\rho}}^{\square, \psi} \cong k\left[\left[y_{11}, \hat{y}_{12}, y_{21}\right]\right]\left[\bar{\pi}, \bar{x}_{11}, \bar{x}_{12}, \bar{x}_{21}\right] .
$$

Since $\mathcal{O}=W(k)$ we have $v \in I \backslash I^{2}$ and hence the elements $I_{1}, I_{2}, I_{3}, I_{4}$ are homogeneous of degree 2 , so that

$$
\operatorname{gr}_{I} R \cong k\left[\left[y_{11}, \hat{y}_{12}, y_{21}\right]\right]\left[\bar{\pi}, \bar{x}_{11}, \bar{x}_{12}, \bar{x}_{21}\right] /\left(I_{1}, I_{2}, I_{3}, I_{4}\right),
$$

see [6, Example 5.3]. Because $R$ is noetherian it follows from [6, Corollary 5.5] that it is enough to show that $\operatorname{gr}_{I} R$ is an integral domain.

We define

$$
A:=k\left[\left[y_{11}, \hat{y}_{12}, y_{21}\right]\right]\left[\bar{x}_{11}, \bar{x}_{12}, \bar{x}_{21}, \bar{\pi}\right] /\left(\bar{I}_{1}\right)
$$

and look at the map

$$
\phi: A \rightarrow A\left[\bar{x}_{12}^{-1}\right] /\left(\bar{I}_{2}\right) .
$$

The latter ring is isomorphic to $\left(k\left[\left[y_{11}, \hat{y}_{12}, y_{21}\right]\right]\left[\bar{x}_{11}, \bar{x}_{12}, \bar{x}_{11}^{-1}, \bar{\pi}\right] /\left(I_{2}\right)\right)$ and since $I_{2}$ is irreducible it is an integral domain. So we would be done by showing that $\operatorname{ker}(\phi)=$ $\left(\bar{I}_{2}, \bar{I}_{3}, \bar{I}_{4}\right)$. The inclusion $\left(I_{2}, I_{3}, I_{4}\right) \subset \operatorname{ker}(\phi)$ is clear from (6) and (8). For the other one we consider the fact that

$$
\operatorname{ker}(\phi)=\left\{a \in A: \exists n \in \mathbb{N} \cup\{0\}, b, c, d \in A: \bar{x}_{12}^{n} a=b \bar{I}_{2}+c \bar{I}_{3}+d \bar{I}_{4}\right\} .
$$

To show that $\operatorname{ker}(\phi) \subset\left(I_{2}, I_{3}, I_{4}\right)$, we let $a \in A$ and $n$ be minimal with the property that there exist $b, c, d \in A$ such that

$$
\bar{x}_{12}^{n} a=b \bar{I}_{2}+c \bar{I}_{3}+d \bar{I}_{4} .
$$


If $n=0$ there is nothing to show. Now we assume that $n>0$ and consider the prime ideal $\mathfrak{p}:=\left(\bar{x}_{12}, \bar{v}-\bar{x}_{11}\right) \subset A$ and see that

$$
A / \mathfrak{p} \cong k\left[\left[y_{11}, y_{12}, y_{21}\right]\right]\left[\bar{x}_{11}, \bar{x}_{12}\right]
$$

is a unique factorization domain. We also observe that

$$
\begin{aligned}
& I_{2} \equiv y_{12}\left(\bar{v}+\bar{x}_{11}\right)^{2} \bmod \mathfrak{p}, \\
& I_{3} \equiv y_{12} \bar{x}_{21}^{2} \bmod \mathfrak{p}, \\
& I_{4} \equiv y_{12}\left(\bar{v}+\bar{x}_{11}\right) \bar{x}_{21} \bmod \mathfrak{p} .
\end{aligned}
$$

Modulo $\mathfrak{p}(9)$ becomes

$$
0 \equiv y_{12} b\left(\bar{v}+\bar{x}_{11}\right)^{2}+y_{12} c \bar{x}_{21}^{2}+y_{12} d\left(\bar{v}+\bar{x}_{11}\right) \bar{x}_{21} .
$$

Since $A / \mathfrak{p}$ is a unique factorization domain there are $b_{1}, c_{1} \in A$ such that

$$
\begin{aligned}
y_{12} b & \equiv b_{1} \bar{x}_{21} \bmod \mathfrak{p}, \\
y_{12} c & \equiv c_{1}\left(\bar{v}+\bar{x}_{11}\right) \bmod \mathfrak{p}
\end{aligned}
$$

and we see that

$$
d \equiv-\frac{b_{1} \bar{x}_{21}+c_{1}\left(\bar{v}+\bar{x}_{11}\right)}{2} \bmod \mathfrak{p} .
$$

Hence we can find $b_{2}, b_{3}, c_{2}, c_{3}, d_{1}, d_{2} \in A$ such that

$$
\begin{aligned}
& b=b_{1} \bar{x}_{21}+b_{2} \bar{x}_{12}+b_{3}\left(\bar{v}-\bar{x}_{11}\right), \\
& c=c_{1}\left(\bar{v}+\bar{x}_{11}\right)+c_{2} \bar{x}_{12}+c_{3}\left(\bar{v}-\bar{x}_{11}\right), \\
& d=-\frac{b_{1} \bar{x}_{21}+c_{1}\left(\bar{v}+\bar{x}_{11}\right)}{2}+d_{1} \bar{x}_{12}+d_{2}\left(\bar{v}-\bar{x}_{11}\right) .
\end{aligned}
$$

Substituting this in (9) we get

$$
\begin{aligned}
\bar{x}_{12}^{n} a= & b \bar{I}_{2}+c \bar{I}_{3}+d \bar{I}_{4} \\
= & \bar{x}_{12}\left(b_{2} I_{2}+b_{3} I_{4}+c_{2} I_{3}+d_{1} I_{4}+d_{2} I_{3}\right) \\
& +\frac{1}{2}\left(b_{1}\left(\bar{v}+\bar{x}_{11}\right)+c_{1} \bar{x}_{21}\right) I_{4}+\left(\bar{v}-\bar{x}_{11}\right) c_{3} I_{3} .
\end{aligned}
$$

Modulo $\mathfrak{p}$ we have $b_{1}\left(\bar{v}+\bar{x}_{11}\right)+c_{1} \bar{x}_{21} \equiv 0$ and hence there are $b_{4}, b_{5}, b_{6}, c_{4}, c_{5}, c_{6}$ with

$$
\begin{aligned}
b_{1} & =\bar{x}_{21} b_{4}+\bar{x}_{12} b_{5}+\left(\bar{v}-\bar{x}_{11}\right) b_{6}, \\
c_{1} & =\left(\bar{v}+\bar{x}_{11}\right) c_{4}+\bar{x}_{12} c_{5}+\left(\bar{v}-\bar{x}_{11}\right) c_{6} .
\end{aligned}
$$

Hence we can rewrite (18) to

$$
\bar{x}_{12}^{n} a=\bar{x}_{12} z+\frac{1}{2}\left(b_{4}+c_{4}\right)\left(\bar{v}+\bar{x}_{11}\right)^{2} I_{3}+\left(\bar{v}-\bar{x}_{11}\right) c_{3} I_{3}
$$


for a certain $z \in\left(I_{2}, I_{3}, I_{4}\right)$. So with (21) we see that $b_{4}+c_{4} \equiv 0$ modulo $\mathfrak{p}$ and $c_{3} \equiv 0$ modulo the prime ideal $\mathfrak{p}^{\prime}:=\left(\bar{x}_{12}, \bar{v}+\bar{x}_{11}\right)$. Therefore we can find some $c_{7}, c_{8}, e_{1}, e_{2} \in A$ with

$$
\begin{aligned}
c_{3} & =c_{7} \bar{x}_{12}+c_{8}\left(\bar{v}+\bar{x}_{11}\right), \\
b_{4}+c_{4} & =e_{1} \bar{x}_{12}+e_{2}\left(\bar{v}-\bar{x}_{11}\right) .
\end{aligned}
$$

But since we have $\left(v+x_{11}\right)\left(v-x_{11}\right)=x_{12} x_{21}$ in $A$ we can finally transform $(21)$ to

$$
\bar{x}_{12}^{n} a=\bar{x}_{12} z^{\prime}
$$

for some $z^{\prime} \in\left(I_{2}, I_{3}, I_{4}\right)$ which shows that $\bar{x}_{12}^{n-1} a \in\left(I_{2}, I_{3}, I_{4}\right)$, since $A$ is an integral domain. But this is a contradiction to the minimality of $n$.

Proposition 1. $R$ is reduced and $\mathcal{O}$-torsion free for any choice of $\mathcal{O}$.

Proof. Since $\mathcal{O}$ is flat over $W(k)$ and we have seen in Lemma 3 that

$$
S:=W(k)\left[\left[x_{11}, \hat{x}_{12}, x_{21}, y_{11}, \hat{y}_{12}, y_{21}\right]\right] /\left(I_{1}, I_{2}, I_{3}, I_{4}\right)
$$

is an integral domain, we get an injection

$$
\mathcal{O} \otimes_{W(k)} S \rightarrow \mathcal{O} \otimes_{W(k)} \operatorname{Quot}(S) .
$$

As $S$ is $W(k)$-torsion free by Lemma 3 , we obtain an isomorphism

$$
\mathcal{O} \otimes_{W(k)} \operatorname{Quot}(S) \stackrel{\cong}{\mathcal{O}}\left[\frac{1}{p}\right] \otimes_{W(k)\left[\frac{1}{p}\right]} \operatorname{Quot}(S) .
$$

Since $\mathcal{O}\left[\frac{1}{p}\right]$ is a separable field extension of $W(k)\left[\frac{1}{p}\right]$, we deduce that $\mathcal{O}\left[\frac{1}{p}\right] \otimes_{W(k)\left[\frac{1}{p}\right]}$ $\operatorname{Quot}(S)$ is reduced and $\mathcal{O}$-torsion free.

\section{The multiplicity}

We want to compute the Hilbert-Samuel multiplicity of the ring $R / \pi$ for the given representation

$$
\bar{\rho}: G_{\mathbb{Q}_{p}} \rightarrow \mathrm{GL}_{2}(k), \quad g \mapsto\left(\begin{array}{cc}
1 & \phi(g) \\
0 & 1
\end{array}\right) .
$$

We denote the maximal ideal of $R / \pi$ by $\mathfrak{m}$.

\section{Theorem 2.}

$$
e(R / \pi)=\left\{\begin{array}{l}
1 \text { if } \bar{\rho} \text { is ramified, } \\
2 \text { if } \bar{\rho} \text { is unramified, indecomposable } \\
4 \text { if } \bar{\rho} \text { is split. }
\end{array}\right.
$$

Proof. If we set $J:=y_{12} x_{21}+2 x_{11} y_{11}+x_{12} y_{21}$ we obtain modulo $\pi$ the relations

$$
\begin{aligned}
& I_{2} \equiv-x_{12} J \\
& I_{3} \equiv x_{21} J \\
& I_{4} \equiv x_{11} J
\end{aligned}
$$


We split the proof into three cases as in Remark 1 . If $\bar{\rho}$ is ramified, i.e., $x_{12}$ is invertible, we see as in the proof of Lemma 4 that

$$
\begin{aligned}
R / \pi & \cong k\left[\left[x_{11}, \hat{x}_{12}, x_{21}, y_{11}, \hat{y}_{12}, y_{21}\right]\right] /\left(x_{11}^{2}+x_{12} x_{21}, J\right) \\
& \cong k\left[\left[x_{11}, \hat{x}_{12}, y_{11}, \hat{y}_{12}\right]\right] .
\end{aligned}
$$

Hence it is a regular local ring and therefore $e(R / \pi)=1$.

Let us assume in the following that $\bar{\rho}$ is unramified, i.e., $x_{12}=\hat{x}_{12} \in \mathfrak{m}_{R}$, and we can consider the exact sequence

$$
0 \rightarrow(R / \pi) / \operatorname{Ann}_{R / \pi}(J) \rightarrow R / \pi \rightarrow R /(\pi, J) \rightarrow 0 .
$$

Since $x_{11}, x_{12}, x_{21} \in \operatorname{Ann}_{R / \pi}(J)$, see $(22)-(24)$, we have $\operatorname{dim}\left((R / \pi) / \operatorname{Ann}_{R / \pi}(J)\right) \leq 3$. But $\operatorname{dim} R / \pi=4$ so that $(25)$ gives us $e(R / \pi)=e(R /(\pi, J))$, see [12, Theorem 14.6]. We obtain that

$$
\begin{aligned}
R /(\pi, J) & \cong k\left[\left[x_{11}, x_{12}, x_{21}, y_{11}, \hat{y}_{12}, y_{21}\right]\right] /\left(x_{11}^{2}+x_{12} x_{21}, J\right) \\
& \cong\left(k\left[\left[x_{11}, x_{12}, x_{21}\right]\right] /\left(x_{11}^{2}+x_{12} x_{21}\right)\right)\left[\left[y_{11}, \hat{y}_{12}, y_{21}\right]\right] /(J)
\end{aligned}
$$

is a complete intersection of dimension 4. So if $\mathfrak{q} \subset R /(\pi, J)$ is an ideal generated by four elements, such that $R /(\pi, J, \mathfrak{q})$ has finite length as a $R /(\pi, J)$-module, then these elements form a regular sequence in $R /(\pi, J)$ and $e_{\mathfrak{q}}(R /(\pi, J))=l(R /(\pi, J, \mathfrak{q}))$, see [12, Theorem 17.11]. Besides, if there exists an integer $n$ such that $\mathfrak{q m}^{n}=\mathfrak{m}^{n+1}$, then $e(R /(\pi, J))=e_{\mathfrak{q}}(R /(\pi, J))$, see [12, Theorem 14.13]. So to finish the proof it would suffice to find such an ideal $\mathfrak{q}$.

If $\bar{\rho}$ is indecomposable, i.e., $\phi(\delta)$ is non-zero and therefore $y_{12}$ is a unit in $R$, we can write the equation $J=0$ as

$$
x_{21}=-y_{12}^{-1}\left(2 x_{11} y_{11}+y_{21} x_{12}\right)
$$

and $I_{1}=0$ as

$$
x_{11}^{2}=x_{12} y_{12}^{-1}\left(2 x_{11} y_{11}+y_{21} x_{12}\right)
$$

so that

$$
R /(\pi, J) \cong k\left[\left[x_{11}, x_{12}, y_{11}, \hat{y}_{12}, y_{21}\right]\right] /\left(x_{11}^{2}-x_{12} y_{12}^{-1}\left(2 x_{11} y_{11}+y_{21} x_{12}\right)\right) .
$$

Hence it is clear that $x_{12}, x_{21}, y_{11}, \hat{y}_{12}$ is a system of parameters for $R /(\pi, J)$ that generates an ideal $\mathfrak{q}$ with $\mathfrak{q m}=\mathfrak{m}^{2}$. So we obtain

$$
e_{\mathfrak{q}}(R /(\pi, J))=l(R /(\pi, J, \mathfrak{q}))=l\left(k\left[\left[x_{11}\right]\right] /\left(x_{11}^{2}\right)\right)=2
$$

and hence $e(R / \pi)=2$.

If $\bar{\rho}$ is split, which is equivalent to $x_{12}, y_{12} \notin R^{\times}$, we take $\mathfrak{q}:=\left(x_{12}-x_{21}, x_{12}-\right.$ $\left.y_{12}, x_{12}-y_{21}, y_{11}\right)$ and claim that $\mathfrak{q m}^{2}=\mathfrak{m}^{3}$. If we write $\mathfrak{m}=\left(x_{12}-x_{21}, x_{12}-y_{12}, x_{12}-\right.$ $\left.y_{21}, y_{11}, x_{11}, x_{12}\right)$ we just have to check that $x_{11}^{3}, x_{11}^{2} x_{12}, x_{11} x_{12}^{2}, x_{12}^{3} \in \mathfrak{q m}^{2}$. Therefore it is enough to see that

$$
\begin{aligned}
& x_{11}^{2}=x_{11} y_{11}-\frac{1}{2}\left(x_{12}-y_{12}\right) x_{21}-\frac{1}{2}\left(x_{21}-y_{21}\right) x_{12} \in \mathfrak{m q}, \\
& x_{12}^{2}=-x_{11}^{2}+x_{12}\left(x_{12}-x_{21}\right) \in \mathfrak{m} \mathfrak{q} .
\end{aligned}
$$


Hence

$$
e(R / \pi)=l(R /(\pi, J, \mathfrak{q}))=l\left(k\left[\left[x_{11}, x_{12}\right]\right] /\left(x_{11}^{2}, x_{12}^{2}\right)\right)=4
$$

Corollary 1. If $\bar{\rho}$ is unramified, then the ring $R$ is not Cohen-Macaulay.

Proof. Since $R$ is $\mathcal{O}$-torsion free, $\pi$ is $R$-regular and hence $R$ is CM if and only if $R / \pi$ is CM. In (25) we have constructed a non-zero submodule of $R / \pi$ of dimension strictly less than the dimension of $R / \pi$. It follows from [5, Theorem 2.1.2(a)] that $R / \pi$ cannot be CM.

Proposition 2. $\operatorname{Spec}(R / \pi)$ is geometrically irreducible and generically reduced.

To prove the proposition we need the following lemma. As in the proof of Theorem 2 we define $J:=y_{12} x_{21}+2 x_{11} y_{11}+x_{12} y_{21}$.

Lemma 5. $R /(\pi, J)$ is an integral domain.

Proof. We again distinguish between three cases as in Remark 1. If $\bar{\rho}$ is ramified, i.e., $x_{12}$ is invertible, we have already seen in the proof of Theorem 2 that

$$
\begin{aligned}
R /(\pi, J) & \cong k\left[\left[x_{11}, \hat{x}_{12}, x_{21}, y_{11}, \hat{y}_{12}, y_{21}\right]\right] /\left(x_{11}^{2}+x_{12} x_{21}, J\right) \\
& \cong k\left[\left[x_{11}, \hat{x}_{12}, y_{11}, \hat{y}_{12}\right]\right] .
\end{aligned}
$$

If $\bar{\rho}$ is unramified and indecomposable, i.e., $x_{12}=\hat{x}_{12} \in \mathfrak{m}_{R}, y_{12} \in R^{\times}$we saw that

$$
R /(\pi, J) \cong k\left[\left[x_{11}, x_{12}, y_{11}, \hat{y}_{12}, y_{21}\right]\right] /\left(x_{11}^{2}-x_{12} y_{12}^{-1}\left(2 x_{11} y_{11}+y_{21} x_{12}\right)\right)
$$

which is easily checked to be an integral domain. If $\bar{\rho}$ is unramified and split, i.e., $x_{12}, y_{12} \in \mathfrak{m}_{R}$, let $\mathfrak{n}$ denote the maximal ideal of $R /(\pi, J)$. It is enough to show that the graded ring $\operatorname{gr}_{\mathfrak{n}} R /(\pi, J)$ is a domain. Since $J$ is homogeneous we have

$$
\operatorname{gr}_{\mathfrak{n}} R /(\pi, J) \cong k\left[x_{11}, x_{12}, x_{21}, y_{11}, y_{12}, y_{21}\right] /\left(x_{11}^{2}+x_{12} x_{21}, J\right) \text {. }
$$

We set $A:=k\left[x_{11}, x_{12}, x_{21}, y_{11}, y_{12}, y_{21}\right] /\left(x_{11}^{2}+x_{12} x_{21}\right)$ and have to prove that $(J) \subset A$ is a prime ideal. We look at the localization map $A \stackrel{\iota}{\rightarrow} A\left[y_{21}^{-1}\right]$, which is an inclusion because $y_{21}$ is regular in $A$. This gives us a map $A \stackrel{\bar{\iota}}{\rightarrow} A\left[y_{21}^{-1}\right] /(J)$. Since

$$
A\left[y_{21}^{-1}\right] /(J) \cong k\left[x_{11}, x_{21}, y_{11}, y_{12}, y_{21}, y_{21}^{-1}\right] /\left(x_{11}^{2}-x_{21} y_{21}^{-1}\left(2 x_{11} y_{11}+x_{21} y_{12}\right)\right)
$$

is a domain, we would be done by showing that $\operatorname{ker}(\bar{\iota})=(J)$. We have

$$
\operatorname{ker}(\bar{\iota})=\left\{a \in A: y_{21}^{i} a=b J \text { for some } i \in \mathbb{Z}_{\geq 0}, b \in A: y_{21} \nmid b\right\} \text {. }
$$

But since $\left(y_{21}\right) \subset A$ is a prime ideal and $y_{21}$ does not divide $J$, we see that $i=0$ in all these equations and hence $\operatorname{ker}(\bar{l})=(J)$.

Proof of Proposition 2. Let $\mathfrak{p}$ be a minimal prime ideal of $S:=R / \pi$. It follows from

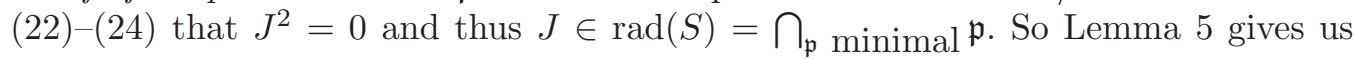
that $J S$ is the only minimal prime ideal of $S$, hence $\operatorname{Spec}(S)$ is irreducible. If we replace the field $k$ by an extension $k^{\prime}$, we obtain the irreducibility of $\operatorname{Spec}\left(S \otimes_{k} k^{\prime}\right)$ analogously, thus $\operatorname{Spec}(S)$ is geometrically irreducible. 
$\operatorname{Spec}(S)$ is called generically reduced if $S_{\mathfrak{p}}$ is reduced for any minimal prime ideal $\mathfrak{p}$. We have already seen that there is only one minimal prime ideal $\mathfrak{p}=J S$. By localizing (25) we obtain $S_{\mathfrak{p}} \cong R /(\pi, J)$. Lemma 5 implies that $S_{\mathfrak{p}}$ is reduced.

\section{Acknowledgment}

This paper was written as a part of my PhD thesis. I want to thank my advisor Prof. Dr. Vytautas Paškūnas for his great support.

\section{References}

[1] G. Böckle, Demuškin groups with group actions and applications to deformations of Galois representations, Compos. Math. 121(2) (2000), 109-154.

[2] C. Breuil and A. Mézard, Multiplicités modulaires et représentations de $\mathrm{GL}_{2}\left(\mathbf{Z}_{p}\right)$ et de $\operatorname{Gal}\left(\overline{\mathbf{Q}}_{p} / \mathbf{Q}_{p}\right)$ en $l=p$, Duke Math. J. 115(2) (2002), 205-310 (with an appendix by Guy Henniart).

[3] - Multiplicités modulaires raffinées, Bull. Soc. Math. France 142(1) (2014), 127-175.

[4] O. Brinon and B. Conrad, CMI summer school notes on p-adic Hodge theory (2009), http://math.stanford.edu/ conrad/papers/notes.pdf.

[5] W. Bruns and J. Herzog, Cohen-Macaulay rings, Cambridge Studies in Advanced Math., 39, Cambridge University Press, Cambridge, 1993, ISBN 0-521-41068-1.

[6] D. Eisenbud, Commutative algebra, Graduate Texts in Math., 150, Springer-Verlag, New York, 1995, ISBN 0-387-94268-8; 0-387-94269-6 (with a view toward algebraic geometry).

[7] M. Emerton and T. Gee, A geometric perspective on the Breuil-Mézard conjecture, J. Inst. Math. Jussieu 13(1) (2014), 183-223.

[8] Y. Hu and F. Tan, The Breuil-Mézard conjecture for non-scalar split residual representations, 2013, arXiv: 1309.1658.

[9] C. Khare and J.-P. Wintenberger, Serre's modularity conjecture II, Invent. Math. 178(3) (2009), $505-586$.

[10] M. Kisin, Potentially semi-stable deformation rings, J. Amer. Math. Soc. 21(2) (2008), 513-546.

[11] - The Fontaine-Mazur conjecture for $\mathrm{GL}_{2}$, J. Amer. Math. Soc. 22(3) (2009), 641-690.

[12] H. Matsumura, Commutative ring theory, 2nd ed., Cambridge Studies in Advanced Math., 8, Cambridge University Press, Cambridge, 1989, ISBN 0-521-36764-6 (translated from the Japanese by M. Reid).

[13] J. Nekovář, On p-adic height pairings, in 'Séminaire de Théorie des Nombres (Paris, 1990-91)', 127-202, Progr. Math., 108, Birkhäuser Boston, Boston, MA, 1993.

[14] V. Paškūnas, On the Breuil-Mézard conjecture, 2012, arXiv:1209.5205.

[15] A. Snowden, Singularities of ordinary deformation rings, 2011, arXiv:1111.3654.

Department of Mathematics, University of Duisburg-Essen, Thea-Leymann-Strasse 9 , 45127 Essen, Germany

E-mail address: fabian.sander@uni-due.de 